\title{
Optimum selection of individual-level neonatal models in place of subject- specific priors for infant diffuse optical tomography
}

Collins-Jones, Liam, Elwell, Clare, Cooper, Robert

Liam H. Collins-Jones, Clare E. Elwell, Robert J. Cooper, "Optimum selection of individual-level neonatal models in place of subject-specific priors for infant diffuse optical tomography," Proc. SPIE 11920, Diffuse Optical Spectroscopy and Imaging VIII, 119200U (9 December 2021); doi: 10.1117/12.2615256 


\title{
Optimum Selection of Individual-Level Neonatal Models in Place of Subject-Specific Priors for Infant Diffuse Optical Tomography
}

\author{
Liam H Collins-Jones ${ }^{\mathrm{a}}$, Clare E Elwell ${ }^{\mathrm{b}}$ and Robert J Cooper ${ }^{\mathrm{a}}$ \\ ${ }^{a}$ DOT-HUB, Biomedical Optics Research Laboratory, Department of Medical Physics and Biomedical Engineering, University College London, \\ London WC1E 6BT, UK \\ ${ }^{b}$ Biomedical Optics Research Laboratory, Department of Medical Physics and Biomedical Engineering, University College London, London \\ WC1E 6BT, UK \\ Authore-mail address liam.jones.14@ucl.ac.uk
}

\begin{abstract}
Diffuse optical tomography relies on anatomical models to simulate light transport. We investigate which cotside measures are best to choose an individual-level head model when subjectspecific data is unavailable for neonatal infants.
\end{abstract}

\section{Introduction}

Diffuse optical tomography (DOT) is an optical imaging technique that requires models of light transport through tissues of the target object in order to reconstruct images. For neuroimaging applications, this requires a model of human head anatomy. Ideally, such a head model would be obtained from a subject's own MRI scan to provide as accurate a representation of anatomical structure as possible. However, it is often not feasible to obtain an MRI scan from every subject; requiring such would undermine the benefits of DOT, notably its tolerance of motion and its ability to be applied in numerous environments, including in the clinical environment and at the infant cotside.

Previous work has looked at producing neonatal head models than can be used in place of infant-specific anatomy for DOT of newborn infants. In this atlas-modelling procedure, known features of the studied infant's anatomy (such as the location of their cranial landmarks) is used to register the age-matched atlas model prior to forward modelling and image reconstruction [1]. Brigadoi et al. [2] produced age-appropriate head models for infants aged from pre-term to term age using averaged MRI data. While these models provide an appropriate population-level model, averaging of structural MRI causes a smoothing of anatomical detail in the model, notably of the cortical surface, so the resulting models are not representative of anatomy at the individual-level.

Recently, we published a database of 215 individual-level neonatal structural head models from infants aged 2944 weeks post-menstrual age (PMA) [3] that were produced primarily for DOT applications using structural MRI data from the Developing Human Connectome Project [4]. Here, we investigate how best to select a head model from this database for a given recruited infant to support real-world DOT data acquisition and image reconstruction. We investigate which characteristics of an infant (which can be feasibly acquired at the cotside) can be used to select a head model that minimises the error incurred relative to using subject-specific anatomy. We quantify this error using anatomical metrics and via simulated optical image reconstruction.

\section{Methods}

\subsection{Anatomical Comparison}

Each head model in the database was chosen in turn to act as the target head model in an approach similar to a leaveone-out analysis. All head models from the database from individuals aged within 2 weeks PMA and with a head circumference within $4 \mathrm{~cm}$ of the target were selected to provide a wide set of potential matches. The 10-5 positions on the scalp surface had been previously computed for each model, which included the 10-20 positions and the cranial landmarks. The origin of these positions was set to be the inion for each individual. Between each potential match and target model the mismatch in head circumference was computed, as was the mean mismatch in 10-20 positions, the mean mismatch of the cranial landmarks positions and the mean scalp surface mesh node-to-node separation. Each selected model was then registered to the target using each of three different registration methods:

- Affine registration using the position of the cranial landmarks. Such data can be collected using a digital positioning system at the cotside, representing a simple scenario where little is known about head shape.

- Affine registration using 10-20 positions. Such data can also be collected from the cotside, representing a case where it is feasible to collect more positioning data.

- Affine Coherent point drift (CPD) registration using a downsampled scalp surface from both the target and selected brain to compute the registration [5]. This represents a cutting-edge method of head registration, 
which is feasible using modern photogrammetry methods to extract a scalp surface from images of infants.

Recent work has employed such a technique in the registration of DOT anatomical models [6].

After registration, the anatomical error between the target (true) model and each registered potential match was calculated in two ways. First, the mean offset between the target and registered model's 10-5 positions were calculated to quantify the mismatch between the true head surface shape and that of the registered model. Second, a neonatal cortical parcellation atlas [7] was used to parcellate the cortical surface and the mean surface distance between equivalent cortical parcellations in the target and the registered models were computed, with these values then averaged across all parcels to yield a single measure of the anatomical difference at the brain-level.

For each of the four mismatch characteristics, the individual with the lowest pre-registration mismatch was determined, and the post-registration mean 10-5 positions offset (i.e. scalp error) and mean cortical parcellation offset (i.e. brain error) was recorded. This was completed in order to determine which of the characteristics that can feasibly obtained from the cotside yield the lowest anatomical error.

\subsection{Optical comparison}

We also aimed to quantify the localization error incurred by using the best-matched model for DOT compared to a model selected purely on the basis of age, and compare both with the gold-standard of subject-specific anatomy. As an example, a single infant aged 39 weeks PMA (the target model) was arbitrarily selected. An optical array consisting of 36 sources and 48 detectors was registered virtually to the target individual using the Homer 2 spring relaxation mechanism [8]. A forward model of photon propagation was produced using Toast++ [9] at $735 \mathrm{~nm}$ and $850 \mathrm{~nm}$. At 12 positions on the cortical surface, Toast++ was used to project simulated perturbations in absorption coefficient of $10 \mathrm{~mm}$ in radius into the channel data space. An image was then reconstructed using these noise-free simulated channel space measurements for the true head model, the model closest in age, and the model selected by the best approach as defined by the anatomical comparison described above.

To enable direct comparison of the resulting images, absorption change images for each head model were transformed to the cortical surface of the target model using Multimodal Surface Matching [10], a spherical registration method. To calculate the centroid of each absorption change image, a weighted averaged of cortical node positions, weighted by absorption changes, was calculated for the true absorption change image, the target model reconstructed image, the transformed metric-matched model reconstructed image, and the transformed age-matched model reconstructed image. The Euclidean separations between the true absorption change centroid and the centroids for each of the other methods were calculated. This was completed for each of the twelve simulated activations at both wavelengths.

\section{Results}

Figure 1a shows the results for the anatomical comparison. For all but one of the anatomical comparisons, the anatomical error for the 10-20 position offset had the lowest discrepancy relative to the best possible error, though the effect is less obvious for the affine CPD registration method using the scalp surface. Affine registration using 10-20 positions also leads to the lowest levels of offset between 10-5 positions post-registration. Figure $1 \mathrm{~b}$ plots the localisation error for each reconstruction at $850 \mathrm{~nm}$, and Figure 1c displays the reconstructed images $850 \mathrm{~nm}$.

\section{Discussion}

This is the first study to conduct an extensive anatomical evaluation of the error incurred in using selected individuallevel models in place of subject-specific anatomical models in neonates. Our results show that selecting a model from our neonatal database on the basis of the subject's $10-20$ position measurements produces a lower error than if a model is selected on the basis of age or head circumference or a variety of other means.

\section{Future work}

This study provides an optimised method for head model selection upon which future image reconstruction involving neonatal infants will be based. In the next few months, we aim to complete our analysis using the entire database, and also include an assessment of the overlap of activated areas in the reconstructed images to better quantify the discrepancies seen between images of the type displayed in Figure 1c. 
a)

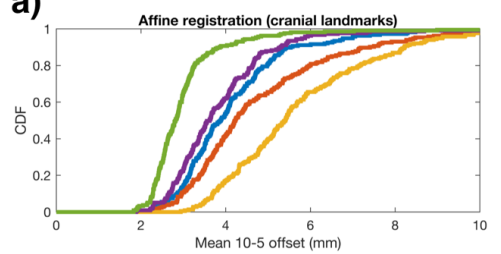

Affine registration (10-20 positions)

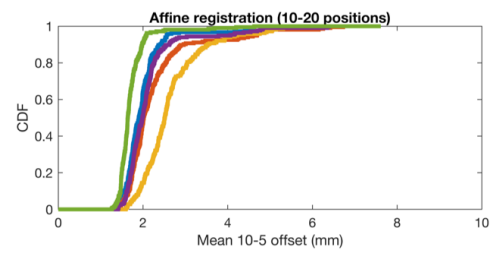

Affine CPD registration (scalp surface)

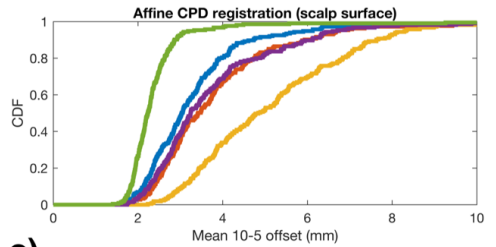

c)

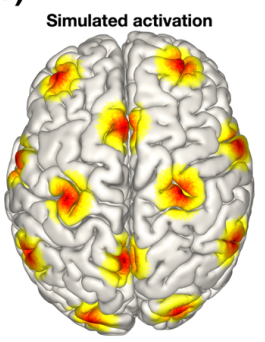

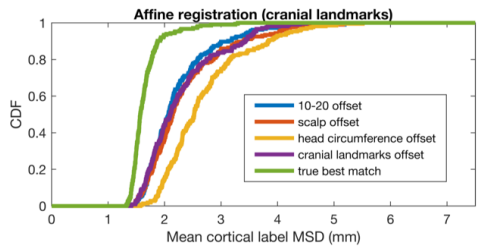
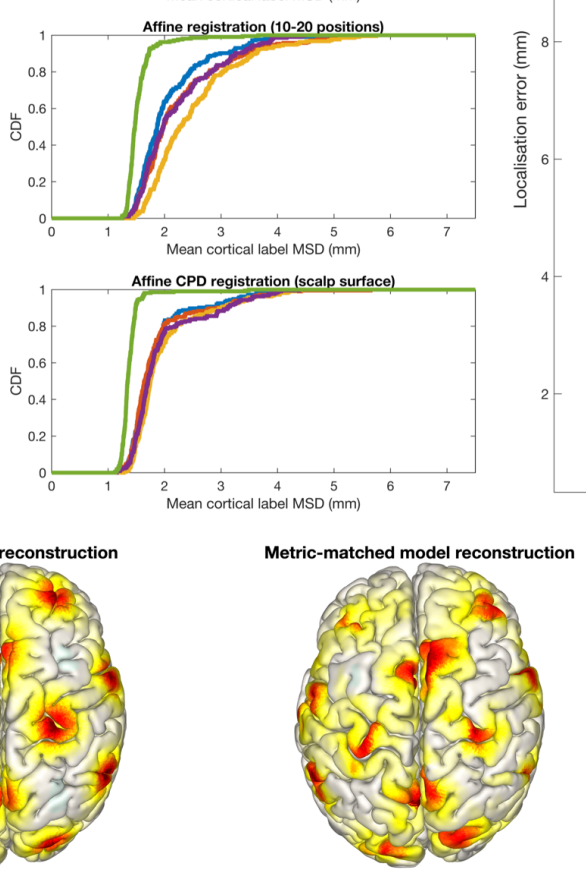

b)

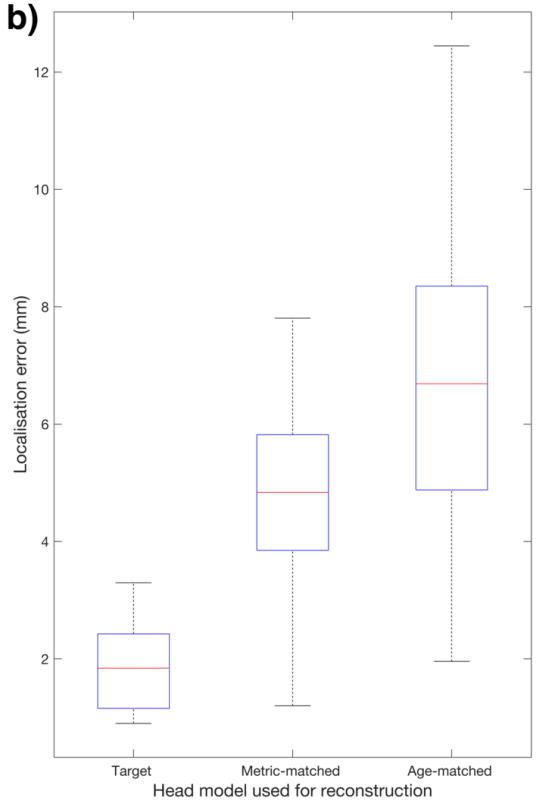

Age-matched model reconstructio

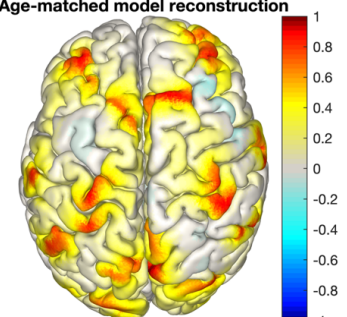

Figure 1. a) Mean 10-5 position offset and mean cortical label mean surface distance (MSD) for the potential match with the lowest mismatch for each characteristic, plotted as a cumulative distribution function. The lowest possible error from the potential matches is shown in green. b) Localisation error of each reconstruction method at $850 \mathrm{~nm}$. c) The simulated absorption changes at $850 \mathrm{~nm}$ are compared to the reconstructed images in each model, shown in the space of the target model.

\section{References}

[1] C. Lee, R.J. Cooper, and T. Austin, "Diffuse optical tomography to investigate the newborn brain," Pediatr. Res., 82, $376-386$ (2017).

[2] S. Brigadoi, P. Aljabar, M. Kuklisova-Murgasova, S.R. Arridge, and R.J. Cooper, "A 4D neonatal head model for diffuse optical imaging of pre-term to term infants," NeuroImage 10, 385-394 (2014).

[3] L.H. Collins-Jones, T. Arichi, T. Poppe, A. Billing, J. Xiao, L. Fabrizi, et al., "Construction and validation of a database of head models for functional imaging of the neonatal brain," Hum. Brain. Mapp., 42, 567-586 (2021).

[4] A. Makropoulos, E.C. Robinson, A. Schuh, R. Wright, S. Fitzgibbon, J. Bozek et al., "The developing human connectome project: A minimal processing pipeline for neonatal cortical surface reconstruction," NeuroImage, 173, 88-112 (2018).

[5] A. Myronenko and X. Song, "Point Set Registration: Coherent Point Drift," in IEEE Transactions on Pattern Analysis and Machine Intelligence, 32(12), 2262-2275 (2010).

[6] E.M. Frijia, A. Billing, S. Lloyd-Fox, E. Vidal Rosas, L. Collins-Jones, M.M. Crespo-Llado et al., "Functional imaging of the developing brain with wearable high-density diffuse optical tomography: A new benchmark for infant neuroimaging outside the scanner environment," NeuroImage, 225, 117490 (2021).

[7] I.S. Gousias, A.D. Edwards, M.A. Rutherford, S.J. Counsell, J.V. Hajnal, D.Rueckert and A. Hammers, "Magnetic resonance imaging of the newborn brain: manual segmentation of labelled atlases in term-born and preterm infants," NeuroImage, 62(3), 1499-1509 (2012).

[8] C.M. Aasted, M.A. Yücel, R.J., Cooper, J. Dubb., D. Tsuzuki, L. Becerra et al., "Anatomical guidance for functional near-infrared spectroscopy: AtlasViewer tutorial," Neurophotonics, 2(2), 020801 (2015).

[9] M. Schweiger and S. Arridge, "The Toast++ software suite for forward and inverse modeling in optical tomography". J. Biomed. Opt. 19(4), 040801 (2014).

[10] E.C. Robinson, S. Jbabdi, M.F. Glasser, J. Andersson, G.C. Burgess, M.P. Harms et al., "MSM: A new flexible framework for multimodal surface matching," NeuroImage, 100, 414-426 (2014).

These results were obtained using data made available from the Developing Human Connectome Project funded by the European Research Council under the European Union's Seventh Framework Programme (FP/2007-2013)/ERC Grant Agreement no. (319456). 